\title{
Hydrogenated Molecules of Carbon as Products of New Pyrolysis Method of Toluene, Xylene and Ethanol
}

\author{
Alexey Kharlamov ${ }^{1}$, Marina Bondarenko, ${ }^{1, *}$, Ganna Kharlamova ${ }^{2}$ \\ ${ }^{1}$ Frantsevich Institute for Problems of Materials Science of NASU, Krzhyzhanovsky St. 3, 03680 Kiev, Ukraine \\ ${ }^{2}$ Taras Shevchenko National University of Kiev, Volodymyrs'ka St. 64, 01601 Kiev, Ukraine \\ *Corresponding Author: dep73@ipms.kiev.ua
}

Copyright $@ 2013$ Horizon Research Publishing All rights reserved.

\begin{abstract}
In contrast to previously known methods of fullerenes and fulleranes producing, firstly the reaction conditions of direct conversion of toluene, xylene and ethanol molecules into molecules of not only fulleranes $\left(\mathrm{C}_{60} \mathrm{H}_{4}-\mathrm{C}_{60} \mathrm{H}_{60}\right.$ and $\left.\mathrm{C}_{70} \mathrm{H}_{10}-\mathrm{C}_{70} \mathrm{H}_{42}\right)$, but also into quasi-fulleranes $\left(\mathrm{C}_{n} \mathrm{H}_{n-6}-\mathrm{C}_{n} \mathrm{H}_{n-2}(\mathrm{n}=20-42)\right)$ were created. The substances containing small molecules of carbon $\mathrm{C}_{3}-\mathrm{C}_{17}$ were synthesized from vapors of toluene, xylene and ethanol under the conditions excluding sublimation of carbon. Firstly the volatile products of thermolysis of hydrogenated molecules of carbon are investigated in all an interval of temperatures $50-750^{\circ} \mathrm{C}$ by means of mass spectrometric method. It is established, that the evolution of hydrogen from given hydrogenated molecules of carbon with so low $(\sim$ $50^{\circ} \mathrm{C}$ ) temperature is starting off. Earlier by anybody yet not synthesized an equiatomic composition fullerane $\mathrm{C}_{60} \mathrm{H}_{60}$ was synthesized, the thin structure of its mass spectrum completely coincides with natural isotope distribution in a molecule $\mathrm{C}_{60} \mathrm{H}_{60}$. The substances synthesized were characterized by temperature-programmed desorption mass spectrometry, matrix-activated laser desorption/ionization mass spectrometry, IR spectroscopy and chemical analysis.
\end{abstract}

Keywords Fullerene, Fulleranes, Quasi-Fullerene, Carbon Molecules, Pyrolysis, Mass Spectra

\section{Introduction}

In 1990, when Kratschmer [1] has developed an arc method of obtaining of fullerene $\mathrm{C}_{60}$ in preparative amounts, Haufler [2] has carried out the reduction of fullerene according to Birch method and has obtained the hydride of fullerene $\mathrm{C}_{60} \mathrm{H}_{36}$ as its first derivative. The experts in the field of hydrogen energy began to place great hopes to create the effective accumulators of hydrogen on a basis of highly hydrogenated fullerenes, which can contain up to 7.7 mass \% of hydrogen. (Though it is necessary to note, that in dodecahedrane $\mathrm{C}_{20} \mathrm{H}_{20}$ [3] a ratio $\mathrm{H} / \mathrm{C}$ the same as in fullerane $\mathrm{C}_{60} \mathrm{H}_{60}$.) It was supposed, that an electron deficient molecule of fullerene can as to hydrogenate at the maximum with formation of the completely hydrogenated molecule, for example, fullerene $\mathrm{C}_{60} \mathrm{H}_{60}$, as in addition to adsorb (approximately 10-15 atoms of hydrogen on a molecule $\mathrm{C}_{60}$ ) hydrogen into a fullerene cage. However numerous and intensive researches to find out more effective methods and reagents for hydrogenating fullerene up to fullerane [4] $\mathrm{C}_{60} \mathrm{H}_{60}$ have not brought expected results. The majority of the methods, developed up today, for synthesizing of fulleranes based extremely on hydrogenation by various reagents of previously synthesized fullerenes, and allow to obtain only partially hydrogenated fullerenes from composition $\mathrm{C}_{60} \mathrm{H}_{2}$ up to composition $\mathrm{C}_{60} \mathrm{H}_{36}$. Attempts to obtain hydrides of fullerenes with the large contents of hydrogen were indispensable finished by the essential complication of process of hydrogenation. So, highly hydrogenated fullerane $\mathrm{C}_{60} \mathrm{H}_{44}$ (with an impurity of minor fractions of composition $\mathrm{C}_{60} \mathrm{H}_{46}-\mathrm{C}_{60} \mathrm{H}_{48}$ ) [5] was obtained only with the usage of a two-stage method: fullerane $\mathrm{C}_{60} \mathrm{H}_{36}$ synthesized under a Birch-Huckel's method at the second stage is additionally hydrogenated under Benkeser's method (by means of reduction it by lithium in ethylenediamine at presence of tert-buthanol $(t-\mathrm{BuOH}))$. A mixture of fulleranes of $\mathrm{C}_{60} \mathrm{H}_{48}-\mathrm{C}_{60} \mathrm{H}_{50}$ composition is synthesized by a liquid-phase (in toluene solution) catalytic $\mathrm{C}_{60}$ hydrogenation (by hydrogen) method under the pressure $12 \mathrm{MPa}$ and at the presence of metal - carbon $(5 \% \mathrm{Ru} / \mathrm{C})$ catalyst [6], that, naturally, reduces an output of fullerane. According to [7] the mixture of $\mathrm{C}_{60} \mathrm{H}_{44}-\mathrm{C}_{60} \mathrm{H}_{52}$ fullerene hydrides can be prepared during 30-90 minutes under terms of direct hydrogenation of fullerite under the ultra-high (2 $\mathrm{GPa})$ pressure and increased $\left(>450^{\circ} \mathrm{C}\right)$ temperatures.

The maximum degree of hydrogenation of fullerene C60 up to composition $\mathrm{C}_{60} \mathrm{H}_{60}$ [4] is reached only at the usage of super-high pressure (9.8 $\mathrm{MPa}$ ) and during long (more than 10 hours) heat treatment at temperatures above $600^{\circ} \mathrm{C}$. However at such severe conditions of hydrogenation there is practically complete destruction of fullerene cage with the formation of a great number of smaller fragments $\mathrm{C}_{59} \mathrm{H}_{\mathrm{x}}$, 
$\mathrm{C}_{58} \mathrm{H}_{\mathrm{x}}, \mathrm{C}_{57} \mathrm{H}_{\mathrm{x}}$ etc. The saturation of destroyed $\mathrm{C}-\mathrm{C}$ bonds in any of fragments $\mathrm{C}_{60-\mathrm{y}} \mathrm{H}_{\mathrm{x}}$ by hydrogen creates only illusion of obtaining of equiatomic composition of fullerane $\mathrm{C}_{60} \mathrm{H}_{60}$.

However, rather high $\left(>400^{\circ} \mathrm{C}\right)$ temperature of fulleranes dehydrogenation has appeared the main obstacle for successful usage of "fullerene-fullerane" system in hydrogen energy. This fact can be quite predicted basically because of high strength of $\mathrm{C}-\mathrm{H}$ bonds comparable with strength of $\mathrm{C}-\mathrm{C}$ bonds. Probably therefore dodecahedrane $\mathrm{C}_{20} \mathrm{H}_{20}$ as steady hydride of fullerene $\mathrm{C}_{20}$, synthesized by Paquette [3] three years prior to opening of fullerene $\mathrm{C}_{60}$ (and 8 years prior to obtaining of the first fullerane $\mathrm{C}_{60} \mathrm{H}_{36}$ ) and containing also $\sim 7.7$ mass $\%$ hydrogen, so steadfast interest of the researchers for some reason has not. Already first experiences on measurement of pressure of allocated gas in process of thermolysis of fullerene hydride in the closed chamber have shown [8], that the dehydrogenation (the composition of volatile products of thermolysis was not investigated) fullerane $\mathrm{C}_{60} \mathrm{H}_{36}$ in crystalline form begins only at temperatures above $400^{\circ} \mathrm{C}$. Probably, therefore it is not enough of researches devoted to study of process of fulleranes thermolysis, though the detailed study of thermal stability of fulleranes is extremely important for understanding of the mechanism of preferable formation of hydrogenated small carbon molecules in comparison with the appropriate molecules of carbon. So Kroto [4] was assumed, what molecules $\mathrm{C}_{\mathrm{n}} \quad($ where $20<\mathrm{n}<60$ ) not containing adjacent pentagons, can be formed mainly as them hydrogenated molecules.

According to the data of the thermogravimetric analysis [9] the intensive loss of mass of samples of fullerene hydride $\mathrm{C} 60 \mathrm{H} 36$ essentially depends on a method of their preparation. So, the dehydrogenation of fullerane $\mathrm{C}_{60} \mathrm{H}_{36}$ prepared by reduction fullerene or by dihydroanthracene, or according to Birch method at temperatures 510 and $380^{\circ} \mathrm{C}$ accordingly is begun. Because the loss of mass of researched samples appreciablly exceeded the contents in them of hydrogen, the assumption [9] was made, that at thermolysis of fullerane there is an allocation not only hydrogen, but also hydrocarbons (for example, methane) formed during partial destruction of fullerene hydride. However products of decomposition of fullerane $\mathrm{C}_{60} \mathrm{H}_{36}$, in particular, the composition of volatile products it thermolysis, in [9] was not investigated. In [10] by a method temperature-programmed desorption (TPD) was shown, that the destruction of a carbon skeleton at decomposition of deuterofullerite $\mathrm{C}_{60} \mathrm{D}_{19}$, obtained deuteration $\mathrm{C}_{60}$, also at $400^{\circ} \mathrm{C}$ is begun. By the authors [10] the gaseous products of desorption were investigated only at two $\left(400\right.$ and $\left.500^{\circ} \mathrm{C}\right)$ temperatures. Thus, all peaks on mass spectra (EI) of a gaseous phase above deuterofullerite were interpeted as peaks of deuterium and fragments of deuterocarbons. However, such conclusions contradict results of researches of carbon materials $[11,12]$ by a TPD method, including fullerenes [12,13], according to which in volatile products of fullerenes desorption in a wide range of temperatures (up to $800^{\circ} \mathrm{C}$ ) also $\mathrm{CO}_{2}, \mathrm{CO}$ and water are present. Therefore peak with $\mathrm{m} / \mathrm{z} 18$, found out by the authors [10] in desorption mass spectra EI at temperatures 400 and $500^{\circ} \mathrm{C}$, can belong as a molecular ion of water $\mathrm{H}_{2} \mathrm{O}^{+}$, so to a fragment deuteromethane $\left[\mathrm{CD}_{2}\right]^{+}$, and the peak with $\mathrm{m} / \mathrm{z} 28$ can correspond to a molecular ion $\mathrm{CO}^{+}$or fragment $\left[\mathrm{C}_{2} \mathrm{D}_{2}\right]^{+}$.

We believe, that the composition of gaseous products of fullerenas thermolysis probably is correct for analyzing only on the basis of desorption mass spectra which have been written down in all an investigated range of temperatures. Only by the comparison of all peaks in continuous thermograms with characteristic mass spectra of water, $\mathrm{CO}$, $\mathrm{CO}_{2}$ and alkanes fragments can be allow authentically to identify volatile products of fulleranes thermolysis.

In the present paper the experimental results demonstrating, first of all, the opportunity to obtain the hydrogenated carbon molecules in grams amounts essentially by other (than hydrogenation of fullerenes) method are submitted. This method excludes a technological stage of preliminary synthesis of carbon molecules: fullerenes and quasi-fullerenes alongside with quasi-fulleranes at the special method of heat treatment of vapors of toluene, xylene and ethanol are formed. Earlier in products of a new method of pyrolysis (NMP) of pyridine [14] (and also benzene [15-17]) we found out of a various type of carbon molecules, such as fullerene $\mathrm{C}_{60}$, it hydrides, quasi-fullerenes $\mathrm{C}_{48}$ and $\mathrm{C}_{40}$, as well as small carbon molecules $\mathrm{C}_{3}-\mathrm{C}_{15}$. By means of a mass spectrometric analysis was convincingly shown, that the hydrogen from products of pyrolysis of pyridine [14] and benzene [16] is appreciablly allocated already at processing of their by a laser. Moreover, at thermolysis of the condensed products of NMP of pyridine and benzene [15-17] the allocation of hydrogen on the data of a method of temperature-programmed desorption mass spectrometry already at temperatures less $100^{\circ} \mathrm{C}$ is observed.

The basic attention in represented research also will be given to comparative study by means of a mass spectrometric analysis of gaseous substances (and, first of all, hydrogen) formed at thermal decomposition (in an interval of temperatures $25-750^{\circ} \mathrm{C}$ ) of various condensate products of NMP of vapors of toluene, xylene and ethanol: fulleranes and quasi-fulleranes, as well as hydrogenated small molecules of carbon $\mathrm{C}_{3}-\mathrm{C}_{15}$. The importance of detection and research of ring molecules of carbon consists that the carbon molecules such as $\mathrm{C}_{2}$ and $\mathrm{C}_{3}, \mathrm{C}_{4}$ and $\mathrm{C}_{5}$ together with polyynes $\left(\mathrm{HC}_{\mathrm{n}} \mathrm{N}\right)(\mathrm{n} \leq 11)$ only in the circumstellar medium [18] are found out. In laboratory conditions these carbon molecules only in solid argon about $15 \mathrm{~K}$ [19] were isolated. However, a time of life of such frozen clusters is extremely small $(\sim 10 \mathrm{~ms})$. Even and odd ions of small carbon molecules in laboratory conditions in mass spectra of carbon vapour are detected only.

\section{Materials and Methods}


The new method of pyrolysis as process of fullerenization of molecules of benzene and pyridine in a different kind of carbon molecules (fullerene $\mathrm{C}_{60}$, quasi-fullerenes $\left(\mathrm{C}_{48}, \mathrm{C}_{42}\right.$ and $\left.\mathrm{C}_{40}\right)$ and flat ring molecules $\left(\mathrm{C}_{3}-\mathrm{C}_{15}\right)$ ) was described by us earlier in [14-17]. Main and distinctive from other methods of pyrolysis (continuous flowing and pulse methods of pyrolysis) the feature of NMP is, that the composition of obtained products depends not only on reactionary conditions (temperature, concentration of reagent, speed of a flow of carrier gas), but also the place of localization of these products in reactionary space. At NMP a part of condesed substances and pyrolytic soot are taken out in a gas reactionary flow from a high-temperature $\left(900-1000^{\circ} \mathrm{C}\right)$ zone $\mathrm{A}$ and are located in more low-temperature zones $\mathrm{B}(\sim$ $\left.300^{\circ} \mathrm{C}\right)$ and $\mathrm{C}\left(\sim 70^{\circ} \mathrm{C}\right)$. Depending on temperature in a zone $\mathrm{A}$, concentration and time of stay in this zone of reagents changes not only the composition of products in zones A, B and $\mathrm{C}$, but also their amount.

The investigation of fullerenization of molecules of toluene, $o$-xylene and ethanol in process of pyrolysis of them vapours are carried out on a usual set of a flowing type under reactionary conditions favorable for forming of a product with mainly by hydrogenated molecules of carbon. Results of study of products of heat treatment of vapours of toluene, $o$-xylene and ethanol, located only in zones B and $\mathrm{C}$ here are submitted. Before by us was established [14-17] that in these zones the carbon molecules of a different type and their hydrogenated forms are mainly condensed. In the most high-temperature zone A the pyrolytic soot is located basically.

The products of several (8-10) experiments at a temperature $\mathrm{T} 1$ or $\mathrm{T} 2$ (where $\mathrm{T} 1>\mathrm{T} 2$ ) obtained in each of the zones $\mathrm{B}$ and $\mathrm{C}$ under similar reactionary conditions are mixed up carefully. The prepared mixture of products were exposed to long processing in the beginning by a benzene and then a xylene (as by the stronger solvent of fullerene) for more complete extraction of the condensed substances. The obtained dark-red extracts from products of zones B and C were concentrated. From the concentrated extracts of zones $\mathrm{B}$ and $\mathrm{C}$ by means of addition of ethanol the dark-red and light-yellow powdery products accordingly were deposited.

(For more convenient representation of experimental results the fixed names to prepared powdery samples were entered. The first letter ( $T, X$ or $E$ ) corresponds to the first letter of the name of precursor: (toluene (T), $o$-xylene (X) or ethanol (E). The second letter corresponds to the name of a zone (B or $\mathrm{C}$ ), third number ( 1 or 2 ) in the fixed name corresponds to a temperature $\mathrm{T} 1$ or $\mathrm{T} 2$ in a zone A. For example, the product of the toluene pyrolysis which taken from a zone $\mathrm{B}$ and obtained at a temperature $\mathrm{T} 1$ in a zone $\mathrm{A}$ is designated as follows: TB1.)

Deposited powdery samples by methods chemical and $\mathrm{X}$-ray diffraction analysis, as well as IR spectroscopy were investigated.

The composition of volatile products of thermal decomposition of powdery precipitation was investigated by a method temperature-programmed desorption mass spectrometry (TPDMS). Thermodesorption measurement was carried out on monopole mass spectrometer MX-7304A (Sumy, Ukraine) with impact electron ionization (EI) [20]. The samples at the bottom of molibdenium-quarts ampoule was evacuated at room temperature up to $5 \cdot 10^{-5} \mathrm{~Pa}$. The linear heating of a sample up to $750^{\circ} \mathrm{C}$ was carried out with speed $0.15 \mathrm{~K} \cdot \mathrm{s}^{-1}$ [20]. The volatile thermolysis products passed through a high-vacuum valve $(5.4 \mathrm{~mm}$ in diameter) into the ionization chamber of the mass-spectrometer The ion currents of the desorption and thermolysis products were recorded with a secondary-electron multiplier VEU-6. Mass spectra were registered in a range 1-210 amu.

Some part of powdery samples again dissolved in a toluene. Prepared toluene solutions placed on a metal plate and after evaporation of the solvent studied by a method of matrix-assisted laser (nitrogen, $337 \mathrm{~nm}$ ) desorption/ionization (MALDI) mass spectrometry (Bruker Daltonics flexAnalysis). For the majority detected in mass spectra the clusters the analysis of thin structure of their peaks was realized (as it was detailed described earlier [14, $16,17]$, that has allowed more correctly to present the composition of detected molecules of carbon and them hydrogenated forms.

The FTIR spectra in a reflectance mode were recorded in the range from 4000 to $400 \mathrm{~cm}^{-1}$ with a spectral resolution of $8 \mathrm{~cm}^{-1}$ using a Nexus Nicolet FTIR spectrometer (Thermo Scientific) equipped with a Smart Collector reflectance accessory. Samples under investigation were powdered with $\mathrm{KBr}$ in 1:10 ratio.

\section{Results and Discussion}

\subsection{Mass Spectrometric Study of thermal Stability and the Composition of Gas-Like Products of Thermolysis of Samples Obtained at the Pyrolysis of Toluene, $o$-Xylene and Ethanol}

All powdery samples deposited from benzene-xylene extracts contain according to their chemical analysis a carbon, a hydrogen and oxygen. The contents of oxygen in products of pyrolysis of toluene and o-xylene does not exceed 1.4 mass $\%$ whereas in products of pyrolysis of ethanol reaches 3.1 mass $\%$. The contents of hydrogen in light-yellow samples TC2 (4.1 mass\%), XC2 (3.9 mass\%), EC2 (4.2 mass\%), considerably is more than in dark-red samples TB2 (2.8 mass\%), XB2 (2.5 mass\%) EB2 (2.7 mass\%). In products of a zone $B$ obtained at higher temperature $\mathrm{T} 1$ in a zone A the contents of hydrogen does not exceed 1 mass $\%$. The amount and the state of hydrogen in condensed and deposited by ethanol hydrogen-containing molecules will be estimated from given of thermodesorption researches.

It is established (Fig. 1) that the hydrogen evolution from all of both toluene, and xylene, and ethanol pyrolysis products condensed in zones $\mathrm{C}$ and $\mathrm{B}$ practically in all 
investigated temperature interval $25-750^{\circ} \mathrm{C}$ is observed. The intensive hydrogen evolution from samples of a zone B (for example, TB2) begins already at temperature $350^{\circ} \mathrm{C}$ whereas the process of the dehydrogenation of fulleranes synthesized at hydrogenation of fullerenes (by hydrogen, dihydroanthracene or by Birch method) only at temperatures above $400^{\circ} \mathrm{C}$ is observed $[9,10]$. Is remarkable, that the hydrogen evolution from products of a zone $\mathrm{C}$ (TC2, XC2, EC2) is fixed (Fig. 1) at much lower $\left(50^{\circ} \mathrm{C}\right)$ temperature of the thermolysis. Comparison of the desorption thermograms of hydrogen (Fig. 1) and mass spectra EI of volatile products of thermal decomposition of identical $(3 \mathrm{mg})$ samples of zones $\mathrm{B}$ and $\mathrm{C}$ shows that these samples under the contents of hydrogen essentially are differed. In products of a zone $\mathrm{C}$ contains almost twice of hydrogen more, than in products of a zone B (Fig. 1). It is important to note, that the curves of temperature dependence of intensity hydrogen evolution from products of a zone $\mathrm{B}$ of pyrolysis have two $(\sim 380$ and $\left.700^{\circ} \mathrm{C}\right)$ and from products of a zone $\mathrm{C}$ have three $(\sim 100,380$ and $700^{\circ} \mathrm{C}$ ) distinct maxima. From fulleranes synthesized at hydrogenation of fullerenes the intensive hydrogen evolution at $700^{\circ} \mathrm{C}$ is observed only. Therefore it is possible to assume, that in products $\mathrm{B} 2$ and $\mathrm{C} 2$ together with fulleranes contain also of different type of hydrogenated carbon molecules, of energy of bonds $\mathrm{C}-\mathrm{H}$ in which appreciablly are differed. It is remarkable, that in the hydrogen thermogram of a product of ethanol pyrolysis EC2 (Fig. 1) intensity of the second temperature maximum much higher.

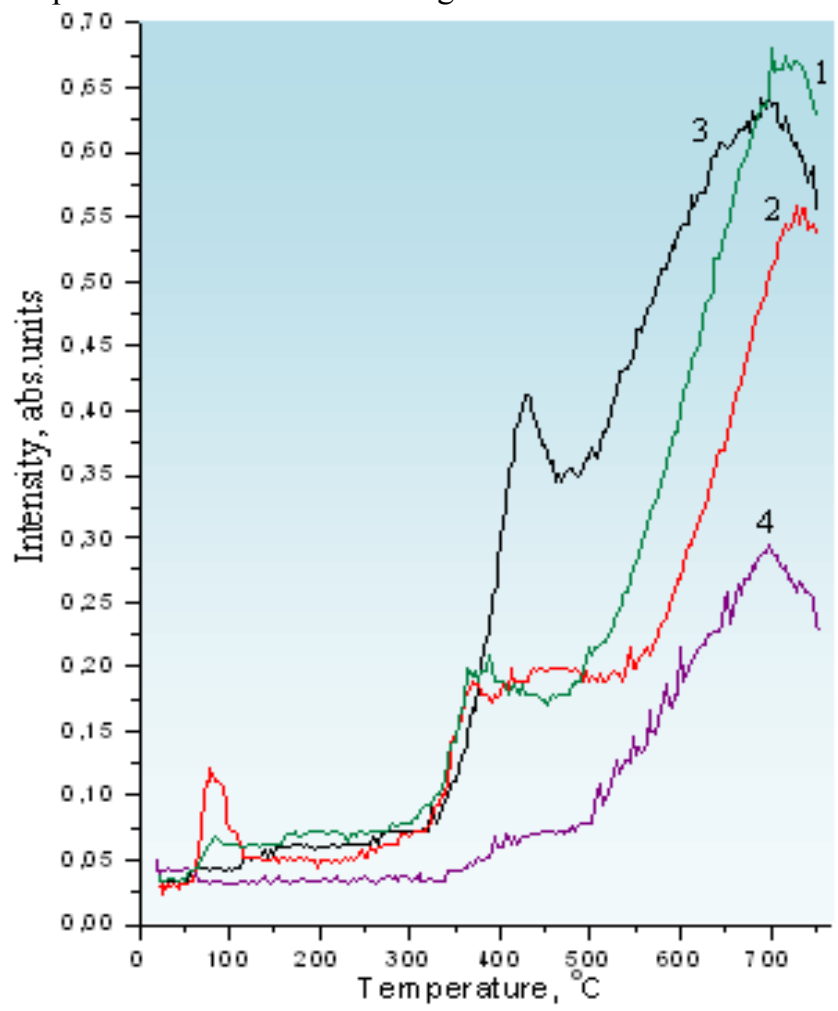

Figure 1. Experimental thermodesorption curves for the 3 amu peaks for samples: 1 - TC1, 2 - XC2, 3 - EC2, 4- TB2

Alongside with hydrogen in a gaseous phase of the thermolysis of all products of the pyrolysis a methane, propane and butane are detected (Fig. 2) the characteristic $100 \%$ by peaks (according to data base of mass spectra National Institute of Standards and Technology USA (NIST)) are ions $\mathrm{CH}_{4}{ }^{+}, \mathrm{C}_{2} \mathrm{H}_{5}{ }^{+}$and $\mathrm{C}_{3} \mathrm{H}_{7}^{+}$with $\mathrm{m} / \mathrm{z} 16,29$ and 43 accordingly.

The hydrocarbons (methane, propane and butane) from products of the pyrolysis, for example, a $o$-xylene $\mathrm{XC} 2$ (Fig. 2) begin to be formed at much lower temperature $\left(\sim 100^{\circ} \mathrm{C}\right)$ than it is observed $\left(400^{\circ} \mathrm{C}\right)$ at dehydrogenation of fulleranes obtained at fullerenes hydrogenation [10]. Thus it is important to note, that the temperature maxima of the most intensive allocation of various hydrocarbons do not coincide. In particular, the maxima of desorption of butane and propane as can be seen from the thermograms of a product XC2 (Fig. 2) are observed in an interval of temperatures $350-400^{\circ} \mathrm{C}$ whereas the destruction of a carbon skeleton of usual fulleranes at higher temperatures occurs. It is remarkable, that allocation of butane and propane from products $\mathrm{C} 2$ occurs only up to temperature $450^{\circ} \mathrm{C}$ whereas the allocation of methane proceeds up to $750^{\circ} \mathrm{C}$. Thus it is necessary to note, that the peak with $\mathrm{m} / \mathrm{z} 16$ corresponds not only methane, but also is alongside with peaks with $\mathrm{m} / \mathrm{z} 18$ and 17 characteristic for mass spectrum of water. However the temperature interval of allocation of methane on the thermogram is precisely observed on a deviation of a maximum of peak with $\mathrm{m} / \mathrm{z} 16$ from maxima of peaks with $\mathrm{m} / \mathrm{z} 18$ and $\mathrm{m} / \mathrm{z} 17$.

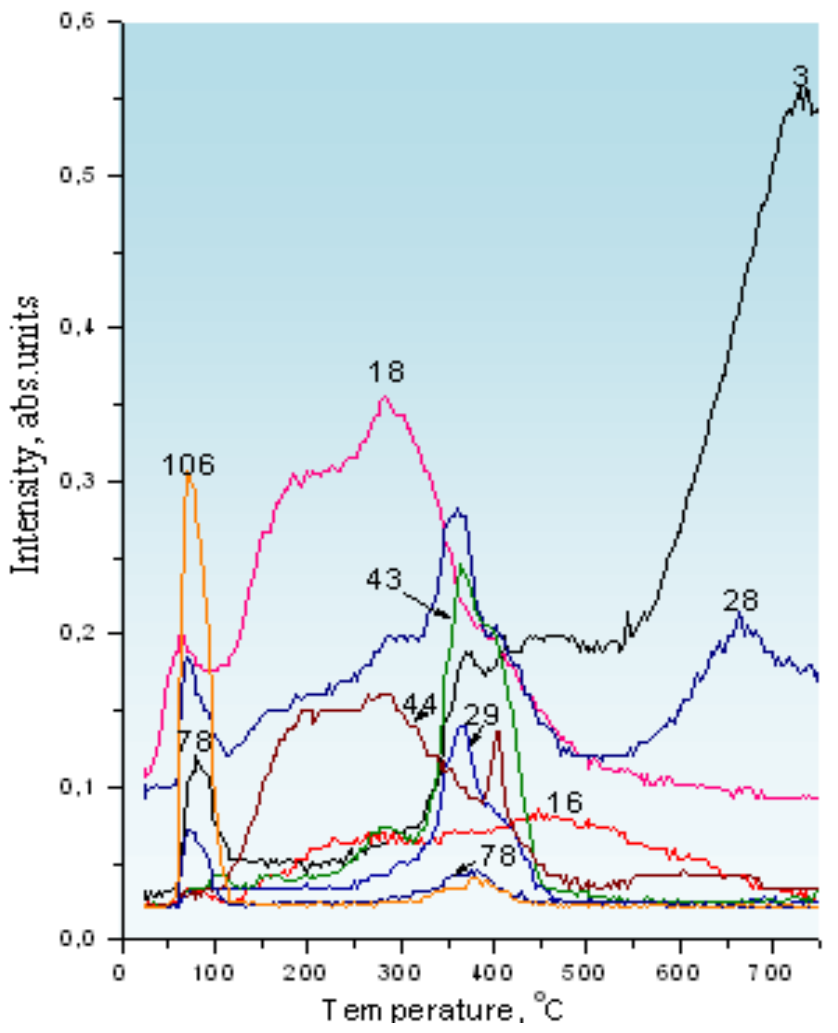

Figure 2. Experimental thermodesorption curves for selected 3, 16, 18, 28, 29, 43, 44, 78 and 106 amu peaks for the product XC2

In all desorption mass spectra EI products of zones B and $\mathrm{C}$ are present also characteristic for thermodesorption of carbon materials [11-13] products $\left(\mathrm{CO}\right.$ with $\mathrm{m} / \mathrm{z} 28$ and $\mathrm{CO}_{2}$ 
with $\mathrm{m} / \mathrm{z} 44$ ) of their oxidations as well as a peak of adsorbed water with $\mathrm{m} / \mathrm{z} 18$ (Fig. 2). Besides an intensive desorption of benzene and xylene (as solvents) submitted on thermograms by peaks of molecular ions with $\mathrm{m} / \mathrm{z} 78$ and 106 accordingly at temperatures $\sim 100^{\circ} \mathrm{C}$ is observed. On thermograms of products of pyrolysis of a zone C (Fig. 2) at $\sim 350^{\circ} \mathrm{C}$ the second weak maximum appropriate to the solvent is seen also. Therefore it is possible to believe, that in process of the deposition by salting-out by ethanol from benzene-xylene solution the part of carbon molecules is deposited as crystal solvates with molecules of the solvent. At the same time rests of ethanol completely leave from all samples at temperatures up to $100^{\circ} \mathrm{C}$.

\subsection{Mass Spectrometric Study of the Composition of Products of Pyrolysis of Toluene, Xylene and Ethanol}

All opening in the field of chemistry of fullerenes are based on mass spectrometric researches: Rofling firstly by mass spectrometric method has found out a complete range of carbon clusters and Kroto, Smalley and Curl have isolated and detected only the cluster $\mathrm{C}_{60}$ as molecule of fullerene. By us toluene solutions of synthesized powdery samples were investigated also by means of MALDI mass spectrometric analysis. Positively and negatively charged clusters registered.

\subsubsection{Mass Spectra of Products of C Zone}

In mass spectra both anions and cations of all three products $\mathrm{C} 2$ obtained at pyrolysis of toluene, xylene and ethanol in zone $\mathrm{A}$ at lower temperature there are intensive peaks of hydrogen (Fig. 3A, inset, Fig. 3B, inset). This fact is unique and in view of presence of hydrogen also in products of NMP of benzene [15-17] and pyridine [14] it is possible to consider its characteristic for products of fullerenization [14-17] of organics. It is remarkable also, that in cations mass spectra of products TC2 (Fig. 3A) and XC2 (Fig. 3B) is present at a range $\mathrm{m} / \mathrm{z} 254-574$ groups of intensive peaks having strict periodicity: the values $\mathrm{m} / \mathrm{z}$ grow serially on 24 or 26 units.

However, in a spectrum of cations of a sample TC2 (Fig. $3 \mathrm{~A})$ this group of peaks has a wider range and up to the value $\mathrm{m} / \mathrm{z} 750$ proceed. The low intensity of highly molecular clusters is quite explained: there is in a spectrum too high intensity of much easier clusters. The spectra of the high resolution of peaks with the values $\mathrm{m} / \mathrm{z} 722$ and 750 correspond to isotope distribution of carbon for fulleranes of compositions $\mathrm{C}_{60} \mathrm{H}_{2}$ and $\mathrm{C}_{60} \mathrm{H}_{30}$ accordingly. Increased intensity of desorption of hydrogen from a product TC2 (Fig.
1) at temperatures $650-750^{\circ} \mathrm{C}$ can be caused by presence at this sample of fullerenes hydrides with high temperature of dehydrogenation.

In spectra of the high resolution it is possible to see (Fig. 4), that the ratios of intensities of peaks ${ }^{12} \mathrm{C}_{\mathrm{n}}$ and ${ }^{12} \mathrm{C}_{\mathrm{n}-1}{ }^{13} \mathrm{C}$ of ions in group of peaks c $254-574 \mathrm{~m} / \mathrm{z}$ correspond to natural isotope distribution of carbon for hydrogenated quasi-fullerenes (quasi-fulleranes) of compositions $\mathrm{C}_{\mathrm{n}} \mathrm{H}_{\mathrm{m}}$ (were $20 \leq n \leq 46$ and $14 \leq m \leq 22$ ). For example, intensity of peaks ${ }^{12} \mathrm{C}_{\mathrm{n}-1}{ }^{13} \mathrm{C}$ of molecules with $\mathrm{m} / \mathrm{z} 326$ (Fig. 4A), 350 (Fig. 4B) and 376 (Fig. 4C) practically coincide with intensity of peaks ${ }^{12} \mathrm{C}_{\mathrm{n}-1}{ }^{13} \mathrm{C}$ counted for molecules $\mathrm{C}_{24} \mathrm{H}_{14}$, $\mathrm{C}_{28} \mathrm{H}_{14}$ and $\mathrm{C}_{30} \mathrm{H}_{16}$ accordingly. At the same time peaks ${ }^{12} \mathrm{C}_{\mathrm{n}-2}{ }^{13} \mathrm{C}_{2}$ of these molecules considerably exceed counted values of isotope distribution of carbon that can testify to detecting in spectra more hydrogenated molecules, in particular, the compositions $\mathrm{C}_{20} \mathrm{H}_{14}-\mathrm{C}_{46} \mathrm{H}_{22}$ accordingly.
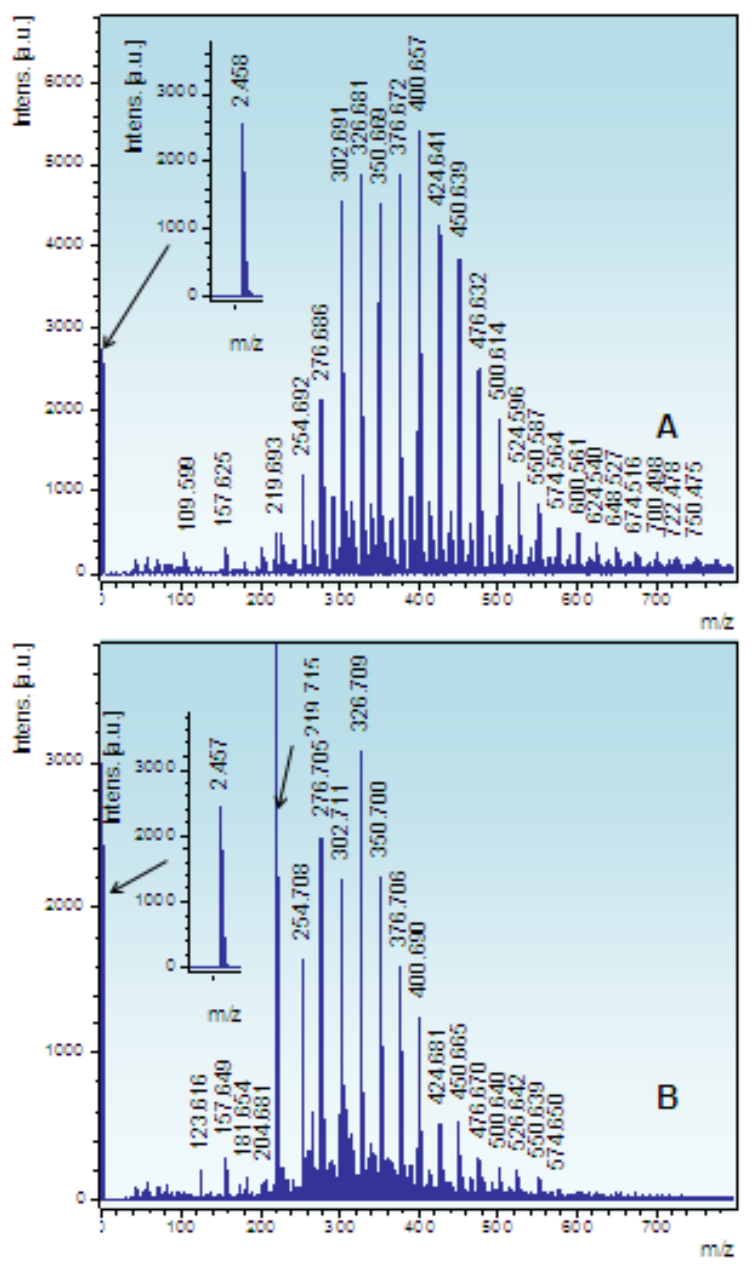

Figure 3. Cations mass spectra of the products TC2 (Part A) and XC2 (Part B) with $\mathrm{m} / \mathrm{z} 1-4$ region in the insets 

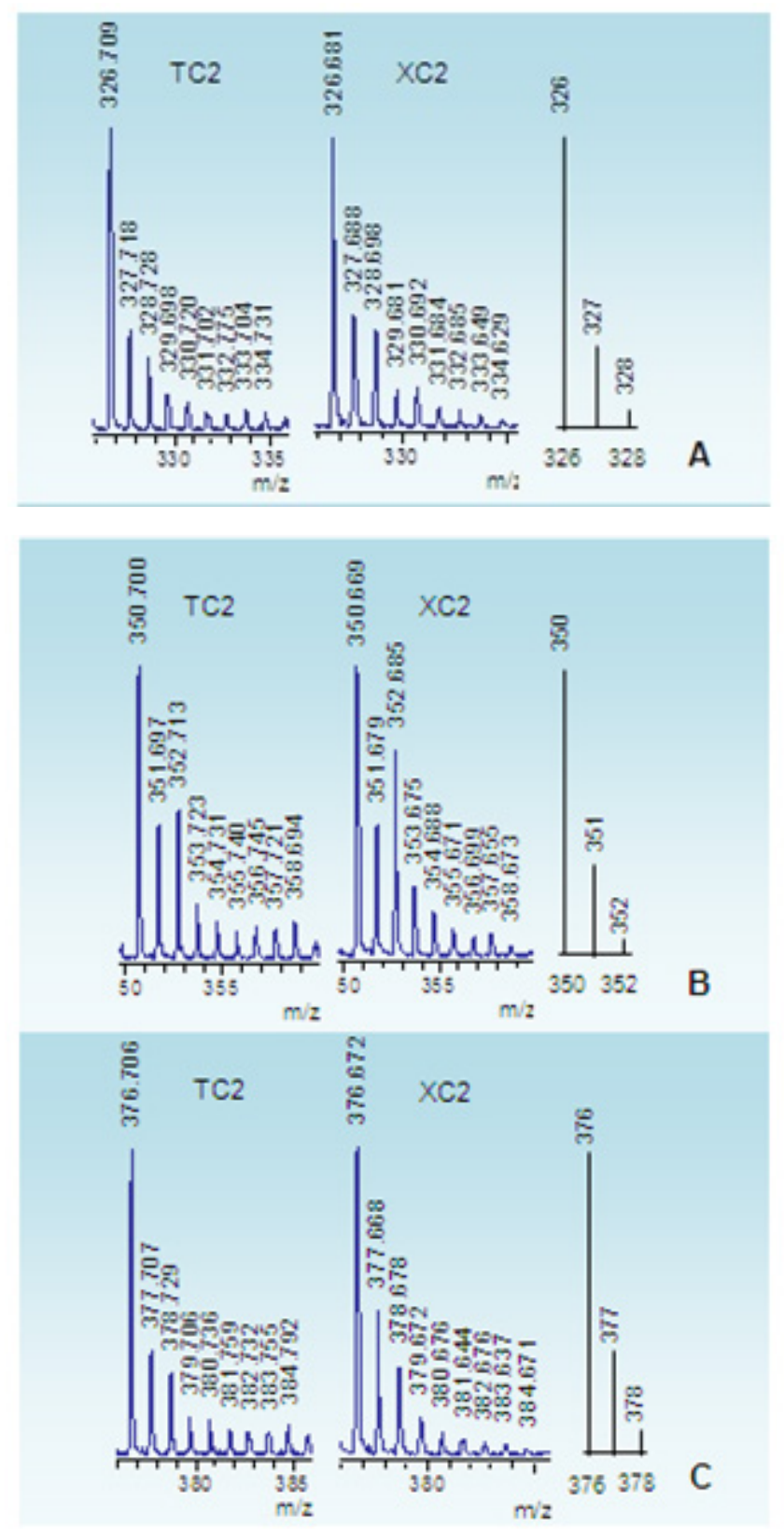

Figure 4. Expansions around $\mathrm{m} / \mathrm{z} 326$ (Part A), 350 (Part B) and 376 (Part C) peaks in the cations mass spectra of the products $\mathrm{TC} 2$ and $\mathrm{XC} 2$ and the calculated isotope mass ratio for $\mathrm{C} 26 \mathrm{H} 14, \mathrm{C} 28 \mathrm{H} 14$ and $\mathrm{C} 30 \mathrm{H} 16$ molecules respectively

On examples of thin spectra submitted on Figure. 4 it is possible distinctly to see, that each of the basic peaks actually consists of several of less intensive peaks, which can be characteristic and for more highly hydrogenated molecules. It is possible to see, that the peaks with $\mathrm{m} / \mathrm{z} 326$, 350 and 376 can correspond to a range of quasi-fulleranes of compositions $\mathrm{C}_{26} \mathrm{H}_{14}-\mathrm{C}_{26} \mathrm{H}_{22}, \quad \mathrm{C}_{28} \mathrm{H}_{14}-\mathrm{C}_{28} \mathrm{H}_{22} \quad$ and $\mathrm{C}_{30} \mathrm{H}_{14}-\mathrm{C}_{30} \mathrm{H}_{24}$ accordingly. It is remarkable, that in a spectrum of negative ions the peaks appropriate hydrogenated quasi-fullerenes absent in general. Only cluster with $\mathrm{m} / \mathrm{z} 326\left(\mathrm{C}_{26} \mathrm{H}_{14}\right)$ is detected as the most intensive peak in positive and negative spectra. Hence, quasi-fullerenes in a reactionary condition NMP are capable to hydrogenate more than on $50 \%$.

\subsubsection{Mass Spectra of Products of B Zone}

The values $\mathrm{m} / \mathrm{z}$ of the most intensive peaks in mass spectra of negative ions of all products of zone B (TB1 (Fig. 5A), $\mathrm{XB1}$ and EB1 (Fig. 5B)) obtained at higher temperature (T1) in a zone A do not exceed 326 basically.

Thus periodicity of change of the value $\mathrm{m} / \mathrm{z}$ among easiest clusters $(36,48,60,72,84,96,108,120,132,144,156,180$ and 204) in this group peaks make 12 units. In mass spectrum of anions of a product TB1 this range of peaks by cluster with $\mathrm{m} / \mathrm{z} 228$ is finished. Let's note that similar periodicity in the change of the values $\mathrm{m} / \mathrm{z}$ of clusters in spectra of anions in products NMP of vapours of benzene [16, 17] and pyridine [14] was found out also. We believe, that the small carbon molecules $\mathrm{C}_{3}, \mathrm{C}_{4}, \mathrm{C}_{5}, \mathrm{C}_{6}, \mathrm{C}_{7}, \mathrm{C}_{8}, \mathrm{C}_{9}, \mathrm{C}_{10}, \mathrm{C}_{11}, \mathrm{C}_{12}, \mathrm{C}_{13}$ $\mathrm{C}_{15}, \mathrm{C}_{17}$ and $\mathrm{C}_{19}$ appropriate to the above mentioned values $\mathrm{m} / \mathrm{z}$ clusters in products of pyrolysis of aromatic hydrocarbons, and ethanol are detected. In such case, the formation of carbon molecules is fulfilled at temperatures ( $~$ $1000^{\circ} \mathrm{C}$ ) excluding evaporation of carbon.
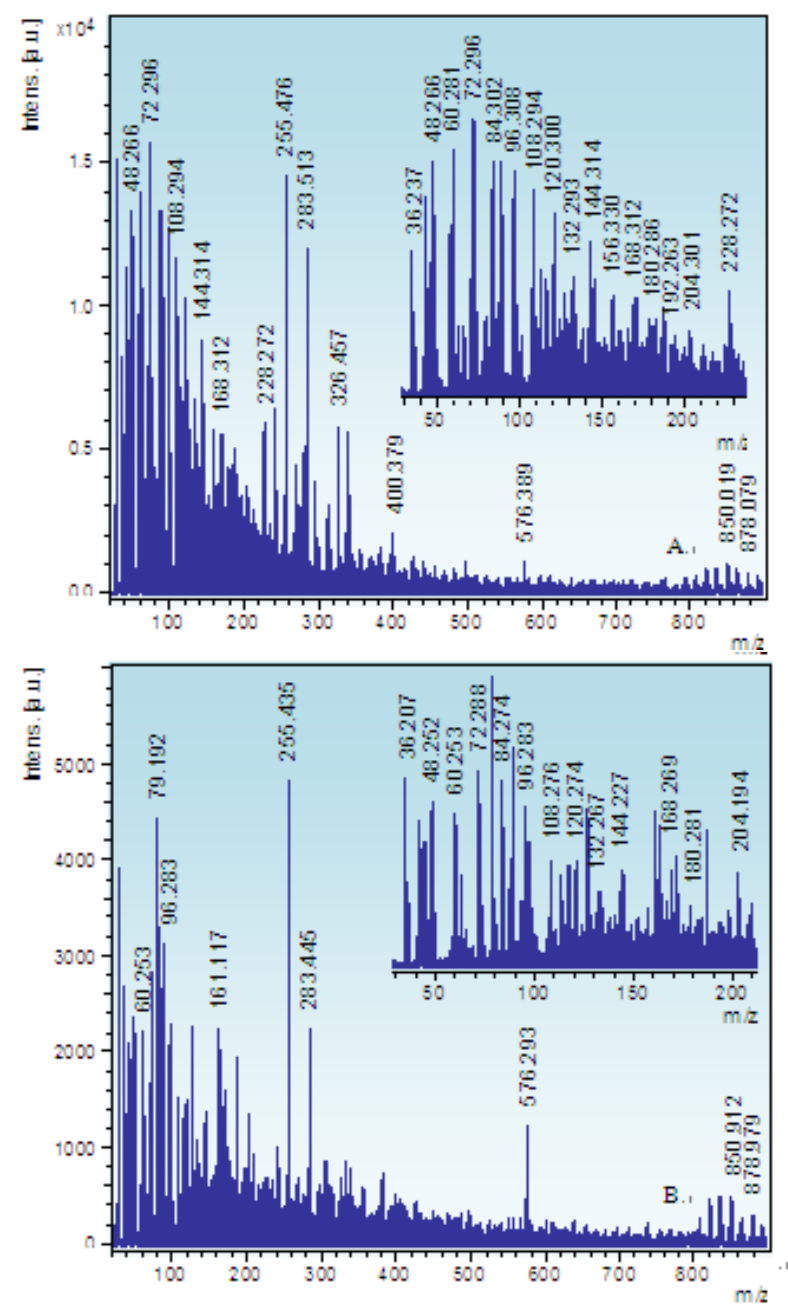

Figure 5. Anions mass spectra of the products TB1 (Part A) and EB1 (Part B) with $\mathrm{m} / \mathrm{z}$ 30-230 regions in the insets

According to thin structure of peaks appropriate small carbon clusters, for example, with $\mathrm{m} / \mathrm{z} 72$ and 84 (Fig. 6), it 
is possible to assume, that both even $\left(\mathrm{C}_{2 \mathrm{n}}\right)$, and odd $\left(\mathrm{C}_{2 \mathrm{n}+1}\right)$ molecules of carbon are partially hydrogenated, as the ratios in natural isotope distribution of carbon in such molecules are overestimated a little. Besides, the even molecules $\left(\mathrm{C}_{2 \mathrm{n}}\right)$ in the greater degree, than odd $\left(\mathrm{C}_{2 \mathrm{n}+1}\right)$ can be hydrogenated.

It is possible also, that these small carbon molecules are products laser destruction of other larger molecules of carbon. However in mass spectra of products B1 are absent both peak of fullerene $\mathrm{C}_{60}$, and peaks characteristic for clusters $\mathrm{C}_{58}$ and $\mathrm{C}_{56}$, as indispensable companions of laser destruction $\mathrm{C}_{60}$. It is possible also to assume, that small carbon clusters $\mathrm{C}_{3}-\mathrm{C}_{17}$ and them hydrogenated forms can be products of the destruction, in particular, quasi-fullerenes or quasi-fulleranes.
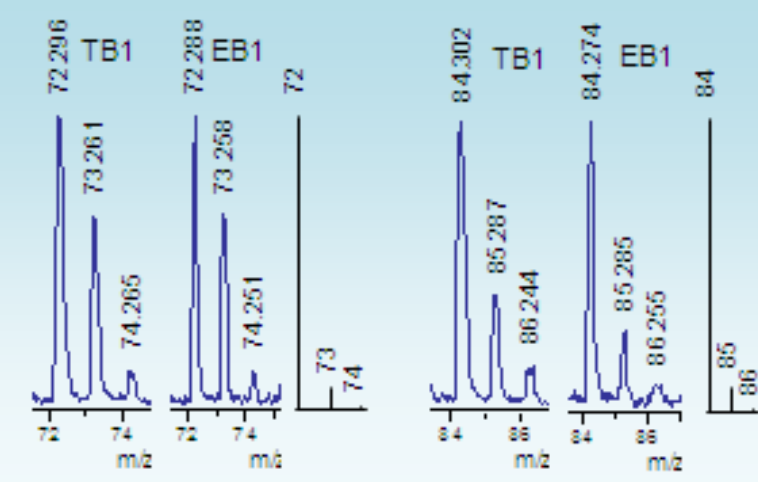

Figure 6. Expansions around $\mathrm{m} / \mathrm{z} 72$ and 84 peaks in the anions mass spectra of the products TB1 and EB1 and the calculated isotope mass ratio for $\mathrm{C}_{6}$ and $\mathrm{C}_{7}$ molecules respectively

At mass spectra of anions of all investigated products of pyrolysis there is a most intensive peak with $\mathrm{m} / \mathrm{z} 255$ which can correspond to anion $\left[\mathrm{C}_{21} \mathrm{H}_{2}-\mathrm{H}\right]^{-}$. This deprotonated ion, probably, at a laser irradiation of minimally a hydrogenated molecule $\mathrm{C}_{21} \mathrm{H}_{2}$ is formed. In mass spectra of cations of products of a zone B (Fig. 3B) (as well as of products of a zone $\mathrm{C}$ (Fig. 3A)) is present one of the most intensive peak with $\mathrm{m} / \mathrm{z} 326$. As follows from thin structure of this peak (Fig. $4 \mathrm{~A}$ ) the value $\mathrm{m} / \mathrm{z} 326$ most full corresponds quasi-fullerane $\mathrm{C}_{26} \mathrm{H}_{14}$.

By distinctive feature of products of pyrolysis of ethanol and toluene is that at mass spectra of negative ions only of products TB1 and EB1 distinctly there are peaks with $\mathrm{m} / \mathrm{z}$ 850 and 878 which can correspond to hydrogenated molecules $\mathrm{C}_{70} \mathrm{H}_{10}$ and $\mathrm{C}_{70} \mathrm{H}_{38}$ of large fullerene $\mathrm{C}_{70}$. Besides, in mass spectra of anions of a product EB1 rather intensive peak with $\mathrm{m} / \mathrm{z} 576$ distinctly is seen also. This cluster firstly was found out by us in mass spectra of products of NMP and benzene [16, 17], and pyridine [14] and according to structure of its thin spectrum was classified as a new molecule of carbon, quasi-fullerene $\mathrm{C}_{48}$.

It is important to note, that molecules $\mathrm{C}_{3}-\mathrm{C}_{17}$ (and them hydrogenatd forms) in mass spectra of cations of all investigated products of pyrolysis of toluene, o-xylene and ethanol are not shown. As earlier by us was established [14,
$16,17]$ the small carbon molecules most precisely are detected just as anions. Fullerene $\mathrm{C}_{60}$ and especially easy products (small carbon clusters) of it destruction are detected mainly as anions [14-17].

In mass spectra as anions (Fig. 7, inset) and cations of products $\mathrm{B} 2$ (synthesized in a zone $\mathrm{A}$ at lower temperature) most intensive is the peak of hydrogen. In mass spectra only of negative ions of products TB2 (Fig. 7) as well as XB1 and EB1 distinctly there is a group of intensive peaks, the values $\mathrm{m} / \mathrm{z}$ and which thin spectra are characteristic for hydrides fullerene $\mathrm{C}_{60}: \mathrm{C}_{60} \mathrm{H}_{4}, \mathrm{C}_{60} \mathrm{H}_{18}, \mathrm{C}_{60} \mathrm{H}_{32}, \mathrm{C}_{60} \mathrm{H}_{46}, \mathrm{C}_{60} \mathrm{H}_{60}$. (In a spectrum of positive ions of products $\mathrm{B} 2$ these fulleranes are not detected at all). Besides, in a spectrum of anions weak peaks of fulleranes $\left(\mathrm{C}_{70} \mathrm{H}_{10}, \mathrm{C}_{70} \mathrm{H}_{42}\right)$ of larger (and less volatile) fullerene $\mathrm{C}_{70}$ are visible also. For example, expansion around peak with $\mathrm{m} / \mathrm{z} 780$ (Fig. 7, inset) is corresponded with calculated isotopic distribution for completely hydrogenated molecule $\mathrm{C}_{60} \mathrm{H}_{60}$ excellently. It is important to note, that distinct detecting in mass spectra of maximum hydrogenated fullerane $\mathrm{C}_{60} \mathrm{H}_{60}$ as anion and thin structure of peak with $\mathrm{m} / \mathrm{z} 780$ precisely confirming its greatest possible composition is found out firstly.



Figure 7. Anions mass spectrum of the product TB2 with $\mathrm{m} / \mathrm{z} 1-4$ region and expansion around $\mathrm{m} / \mathrm{z} 780$ peak and the calculated isotope mass ratio for $\mathrm{C}_{60} \mathrm{H}_{60}$ molecule in the insets

Earlier [7] by a mass spectrometric method were detected only the cations of fulleranes of compositions $\mathrm{C}_{60} \mathrm{H}_{36}-\mathrm{C}_{60} \mathrm{H}_{52}$ obtained by long hydrogenation of fullerene $\mathrm{C}_{60}$ at the increased temperatures and ultra-high pressure. Cations with the large values $\mathrm{m} / \mathrm{z}$ were interpeted as oxyhydrides of fullerene. In particular, the peak with $\mathrm{m} / \mathrm{z} 785$ as the cation $\mathrm{C}_{60} \mathrm{H}_{49} \mathrm{O}^{+}$of fullerene oxyhydride $\mathrm{C}_{60} \mathrm{H}_{50} \mathrm{O}$ ) was interpeted. However, as already it was marked above, under such rigid reactionary conditions fullerene cage significantly is collapsed, i.e. the process of defullerenization of a spheroidal molecule was observed. Therefore, the peak with $\mathrm{m} / \mathrm{z} 780$ in mass spectra of such defullerenization product could not be detected basically.

It is important to note, that at a spectrum of anions there is 
also rather intensive peak with $\mathrm{m} / \mathrm{z} 62$, which can correspond to minimally hydrogenated carbon molecule $\mathrm{C}_{5}$.

Thus, the results of mass spectrometric researches convincingly confirm (Fig. 5, 6 and 7) that in reactionary conditions of NMP the process of fullerenization of molecules not only aromatic hydrocarbons but also ethanol is realized: the simultaneous formation of a different type of carbon molecules and them hydrogenated forms is observed. Firstly, molecule of fullerane of an equiatomic composition $\mathrm{C}_{60} \mathrm{H}_{60}$ by a mass spectrometric method in powdery products of pyrolysis of toluene was detected, whereas numerous attempts to obtain fullerane $\mathrm{C}_{60} \mathrm{H}_{60}$ by methods of the hydrogenation of fullerene $\mathrm{C}_{60}$ are still unsuccessful. The fact of formation an equiatomic fullerane $\mathrm{C}_{60} \mathrm{H}_{60}$ from molecules of toluene is especially unique as before about an opportunity of its existence only assumed.

\subsection{Research of IR Spectra of the Condensed Products of Pyrolysis Toluene, O-Xylene and Ethanol}

The structure of IR spectra of samples TB2, XB2 and EB2 taken from the condensed products of pyrolysis accordingly toluene, $o$-xylene and ethanol, basically is similar and in many respects reminds the structure of IR spectra of fulleranes of different compositions prepared by hydrogenation fullerenes. So, the values of the basic absorption bands in IR spectrum, for example, sample TB2 (Fig. 8), are rather close to the values established for fullerane $\mathrm{C}_{60} \mathrm{H}_{36}[2,21,22]$ as well as to calculated value for hypothetical $\mathrm{C}_{60} \mathrm{H}_{60}$ [23] (Table 1). The molecules of partially hydrogenated fullerenes (for example, fullerane $\mathrm{C}_{60} \mathrm{H}_{36}$ ) have smaller symmetry, than fullerene $\mathrm{C}_{60}$ (has only four modes with symmetry $\mathrm{F}_{1 \mathrm{u}}$ ) [23] and fullerane of an equiatomic composition, $\mathrm{C}_{60} \mathrm{H}_{60}$. For $\mathrm{C}_{60} \mathrm{H}_{60} 9$ active modes [23] (Table 1) are calculated, three from which concern to vibrations of $\mathrm{C}-\mathrm{H}$ bonds and 6 correspond to vibrations of fullerene skeleton. Therefore, IR spectra of partially hydrogenated fullerenes have the a little bit greater number of bands, as follows from submitted Table 1 .

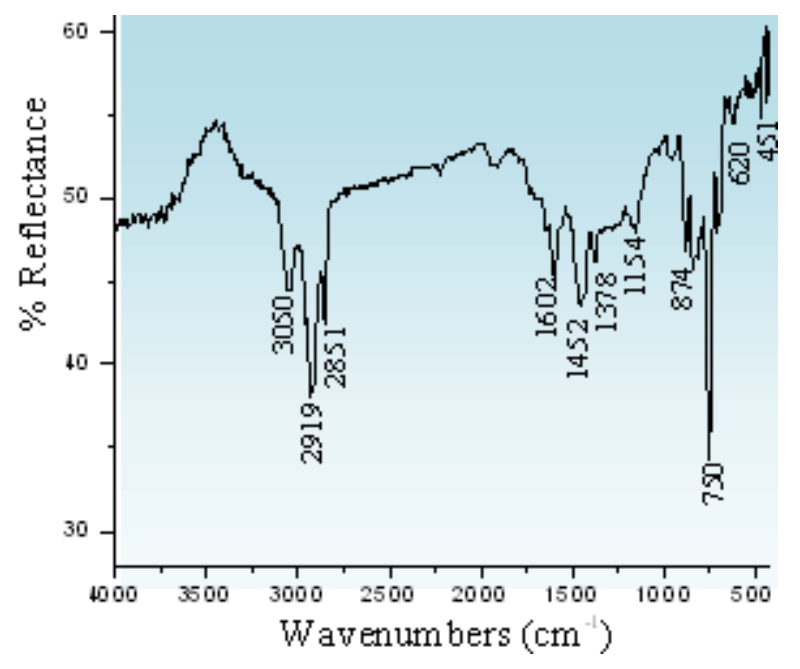

Figure 8. IR spectrum of the product TB2 
Table 1. Comparison of the main absorption bands in the IR spectra of samplesTB1 and C60H36 [2, 21, 22] and calculated [23] for C60H60 values of the absorption bands

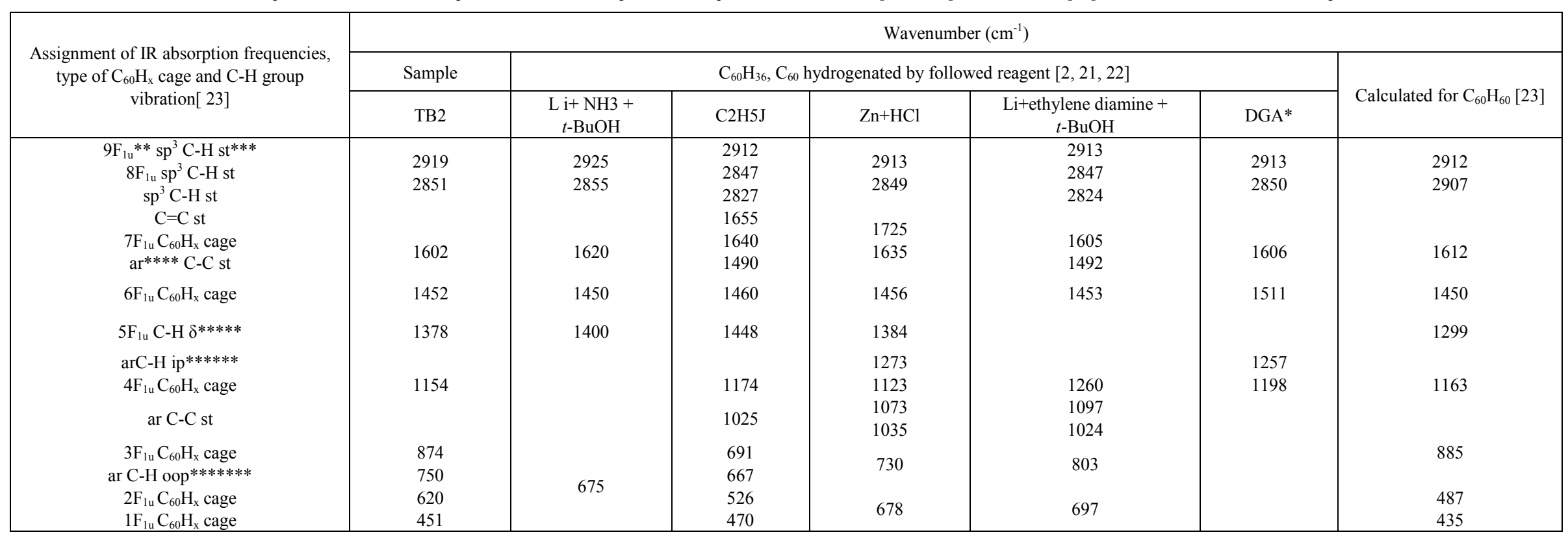

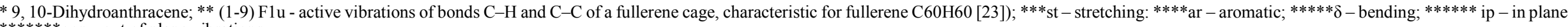
$* * * * * * *$ oop - out of plane vibrations. 
According to structure of IR spectrum of a sample TB2 it is possible to assume, that the products of pyrolysis, in particular, a toluene contain mainly hydrogenated molecules of carbon. The absorption bands in IR spectrum of a sample TB2 responsible, probably, for vibrations of the deformed spherical skeleton of carbon molecules (Table 1, Fig. 8) have the values $1602,1452,1154,874,620,451 \mathrm{~cm}^{-1}$. Just such absorption bands of a sample TB2 are closest to counted values of absorption bands of a carbon skeleton of an equiatomic fullerane $\mathrm{C}_{60} \mathrm{H}_{60}$ [23]. Agrees of mass spectrometric researches the condensed products of pyrolysis of toluene contain (Fig. 7) such fulleranes as $\mathrm{C}_{60} \mathrm{H}_{46}, \mathrm{C}_{60} \mathrm{H}_{60}$ и $\mathrm{C}_{70} \mathrm{H}_{36}$. The band at $750 \mathrm{~cm}^{-1}$, observable in IR spectrum TB2, is close to the value 744 and $730 \mathrm{~cm}^{-1}$, which are characteristic for fullerane $\mathrm{C}_{60} \mathrm{H}_{36}$, obtained by hydrogenation fullerene with use $\mathrm{C}_{2} \mathrm{H}_{5} \mathrm{~J}$ [24] и $\mathrm{Zn}+$ conc. $\mathrm{HCl}$ [21] accordingly.

Characteristic for $\mathrm{C}-\mathrm{H}$ bonds the stretching vibrations at 2919 and $2851 \mathrm{~cm}^{-1}$ and the bending vibrations at $1378 \mathrm{~cm}^{-1}$ observable in IR spectrum of a sample TB2 satisfactorily coordinated with IR spectra of fullerene hydrides obtained by different methods [2, 21, 22, 25-31] (Table 1). However, the values of bands $\mathrm{C}-\mathrm{H}$ bonds are especially close for a sample TB2 and fullerane $\mathrm{C}_{60} \mathrm{H}_{36}$ obtained on a method of Birch-Huckel [2] (Table. 1). The weak band at $\sim 3050 \mathrm{~cm}^{-1}$ characteristic for the stretching vibrations of aromatic $\mathrm{C}-\mathrm{H}$ bonds testifies to presence at products of solvated aromatic solvents (benzene and xylene).

On the basis of the analysis of structure of IR spectra of products of pyrolysis both toluene, and $o$-xylene, and ethanol it is possible to make a conclusion that these spectra practically completely coincide with structure of IR spectra of fulleranes prepared by method of the hydrogenation of fullerenes.

Thus, the submitted experimental results convincingly testify, that the molecules quasi-fulleranes $\left(\mathrm{C}_{20-46} \mathrm{H}_{14-44}\right)$ and fulleranes $\left(\mathrm{C}_{60} \mathrm{H}_{4}-\mathrm{C}_{60} \mathrm{H}_{60}\right.$ and $\left.\mathrm{C}_{70} \mathrm{H}_{8}-\mathrm{C}_{70} \mathrm{H}_{38}\right)$ as well as small carbon molecules $\mathrm{C}_{3}-\mathrm{C}_{17}$ and quasi-fullerene $\mathrm{C}_{48}$ are the basic condensed products of a new method of pyrolysis of vapours of toluene, $o$-xylene and ethanol. The formation of carbon molecules and their hydrogenated forms is fulfilled simultaneously at temperatures excluding evaporation of carbon. In parallel with reactions of polymerization (hydrogenated molecules) and dehydropolymerization (carbon molecules) the reaction of destruction of molecules precursor proceeds. Molecules $\mathrm{C}_{3}-\mathrm{C}_{5}$ at destruction of molecules not only toluene and xylene but ethanol are formed. The formation of fulleranes and quasi-fullerene $\mathrm{C}_{48}$ from ethanol molecule convincingly testifies to participation in their growth small carbon clusters.

Hydrogenated carbon molecules it is easy by aromatic hydrocarbon from pyrolytic soot are extracted and by ethanol from the concentrated extracts as red and light yellow powders are deposited. The evolution of hydrogen at thermolysis of hydrogenated carbon molecules synthesized in process of fullerenization of aromatic hydrocarbons and ethanol already at temperature $50^{\circ} \mathrm{C}$ is starting off. Their decomposition with formation of methane, propane and butane at lower temperatures also proceeds, than it is observed for usual fulleranes prepared at the hydrogenation of fullerenes.

\section{Conclusion}

1. In contrast to known the methods of pyropysis, the reactionary conditions of direct transformation of molecules toluene, $o$-xylene and ethanol into molecules not only fulleranes $\left(\mathrm{C}_{60} \mathrm{H}_{4}-\mathrm{C}_{60} \mathrm{H}_{60}\right.$ and $\left.\mathrm{C}_{70} \mathrm{H}_{10}-\mathrm{C}_{70} \mathrm{H}_{42}\right)$, but also in molecules of new substances - quasi-fulleranes $\left(\mathrm{C}_{n} \mathrm{H}_{n-6}-\mathrm{C}_{n} \mathrm{H}_{n-2}(\mathrm{n}=20-46)\right)$ are created. In this new method a stage of preliminary synthesis of fullerenes is excluded completely. At the same time, the carbon molecules and, in particular, fullerenes can be intermediate substances in reactions of formation fulleranes.

2. Firstly, by mass spectrometric method in powdery product contained to hydrogenated carbon molecules is detected as anion $\mathrm{C}_{60} \mathrm{H}_{60}{ }^{-}$maximum saturated by hydrogen fullerane $\mathrm{C} 60 \mathrm{H} 60$. An equiatomic composition fullerane $\mathrm{C}_{60} \mathrm{H}_{60}$ was synthesized in process of fullerenization of toluene molecules.

3. The samples of hydrogenated carbon molecules by a new method of pyrolysis in grams amounts are obtained. Significant dehydrogenation of the synthesized samples according to results of mass spectrometric investigation of their thermolysis products at $\sim 50^{\circ} \mathrm{C}$ is starting off, whereas dehydrogenation of usual fulleranes (synthesized by the hydrogenation of fullerenes) only at $400^{\circ} \mathrm{C}$ is observed.

\section{REFERENCES}

[1] W. Kratschmer, L. D. Lamb, K. Fostiropoulos, D. R. Huffman. Solid C60: A New Form of Carbon, Nature, Vol.347, No.6291, 354-358, 1990.

[2] R. E. Haufler, J. Conceicao, L. P. F. Chibante, Y. Chai, N. E. Byrne, S. Flanagan, M. M. Haley, S. C. O’Brien, C. Pan, Z. Xiao, W. E. Billups, M. A. Ciufolini, R. H. Hauge, J. L. Margrave, L. J. Wilson, R. F. Curl, R. E Smalley. Efficient Production of C60 (Buckminsterfullerene), $\mathrm{C} 60 \mathrm{H} 36$, and the Solvated Buckide Ion, The Journal of Physical Chemistry, Vol.94, 8634-8636, 1990.

[3] L. A. Paquette, R. J. Ternansky, D. W. Balogh, G. Kentgen. Total synthesis of dodecahedrane, Journal of the American Chemical Society, Vol.105, No.16, 5446-5450, 1983.

[4] F. Cataldo, S. Iglesias-Groth. Fulleranes: The Hydrogenated Fullerenes, Springer, Dordrecht, 2010.

[5] A. Peera, R. K. Saini, L. B. Alemany, W. E. Billups, M. Saunders, A. Khong, M. S. Syamala, R. J. Cross. Formation, Isolation, and Spectroscopic Properties of Some Isomers of $\mathrm{C} 60 \mathrm{H} 38$, C60H40, C60H42 and C60H44, European Journal of Organic Chemistry, Vol.21, 4140-4145, 2003. 
[6] K. Shigematsu, K. Abe, M. Mitani K. Tanaka. Catalytic Hydrogenation of Fullerenes in the Presence of Metal Catalysts in Toluene Solution, Fullerene Science and Technology. Vol.1, No.3, 309-318, 1993.

[7] A. V. Talyzin, A. Dzwilewski, B. Sundqvist, Y.O. Tsybin, J.M. Purcell, A.G. Marshall, Y.M. Shulga, C. McCammon, L. Dubrovinsky. Hydrogenation of C60 at $2 \mathrm{GPa}$ pressure and high temperature, Chemical Physics, Vol.325, 445-451, 2006.

[8] F. Chen, W. Li, R. Loutfy, R. Murphy, J. Wang. Hydrogen Storage in Fullerenes and in an Organic Hydride, Proceedings of the 1998 U.S. DOE Hydrogen Program Review, USA. 1998.

[9] A. A. Peera, Fullerene hydrides and studies toward the synthesis of fulvalenes. Ph.D.Thesis. Houston. Texas. 2004.

[10] Y. M. Shul'ga, B. P. Tarasov, V. N. Fokin. Deuterofullerenes, Carbon, Vol.41, No.7, 1365-1368, 2003.

[11] M. S. R. Zacharia. Desorption of gases from graphitic and porous carbon surfaces. Ph.D.Thesis. Freie Universität Berlin, Berlin. 2004.

[12] T. Y. Gromovoy, B. B., Palyanytsya, V. A. Pokrovskiy, E. V. Basiuk, V. A. Basiuk. Interaction of thermally pretreated carbon nanomaterials with water vapor, Journal of Nanoscience and Nanotechnology, Vol.4, No.1-2, 77-81, 2004.

[13] M. Wohlers, A. Bauer, T. Belz, T. Rühle, T. Schedel-Niedrig, R. Schlögl. The Mechanism of Oxidation of Fullerenes with Molecular Oxygen, American Chemical Society Symposium Series, 108-112, 1996.

[14] A. Kharlamov, G. A. Kharlamova, M. E. Bondarenko. New products of a new method of pyrolysis of pyridine, Russian Journal of Applied Chemistry, Vol.86, No.2, 183-190, 2013.

[15] A. I. Kharlamov, M. E. Bondarenko, N. V. Kirillova. New method for synthesis of fullerenes and fullerene hydrides from benzene, Russian Journal of Applied Chemistry, Vol.85, No.2, 233-239, 2012.

[16] A. Kharlamov, G. Kharlamova, M. Bondarenko, V. Fomenko. New method of generation of carbon molecules and clusters, Open Journal of Synthesis Theory and Applications, Vol.2. No.1, 38-45, 2013.

[17] A. Kharlamov, G. Kharlamova, M. Bondarenko, V. Fomenko. Joint Synthesis of Small Carbon Molecules (C3-C11), Quasi-Fullerenes (C40, C48, C52) and their Hydrides, Chemical Engineering and Science, Vol.1, No.3, 32-40, 2013.

[18] F. Cataldo. Cyanopolyynes: carbon chains format ion in a carbon arc mimicking the formation of carbon chains in the circumstellar medium, International Journal of Astrobiology, Vol.3, 237 -246, 2004.

[19] Wakabayashi, W. Kratschmer. Carbon Chain Molecules in Cryogenic Matrices. In: Polyynes Synthesis, Properties, and
Applications, Ed. F.Cataldo, Taylor, New York, USA, Chap. 1, 1- 15, 2006.

[20] T. V. Kulik, V. N. Barvinchenko, B. B. Palyanitsa, O. V. Smirnova, V. K. Pogorelyi, A. A. Chuiko. A Desorption Mass Spectrometry Study of the Interaction of Cinnamic Acid with a Silica Surface, Russian Journal of Physical Chemistry A, Vol.81, No.1, 83-90, 2007.

[21] N. F. Goldshleger, A. P. Moravsky. Hydrides of the fullerenes, Uspekhi Khimii, Vol.66, No.4, 353-375, 1997. (Russian)

[22] J. P. Zhang, N. X. Wang, Y. X. Yang. A. G. Yu. Hydrogenation of [60] fullerene with lithium in aliphatic amine, Carbon, Vol.42, No.3, 667-691, 2004.

[23] R. Stoldt, R. Maboudian, C. Carraro. Vibrational Spectra of Hydrogenated Buckminsterfullerene: A Candidate for the Unidentified Infrared Emission, The Astrophysical Journal, Vol.548, 225-228, 2001.

[24] P. A. Dorozhko, A. S. Lobach, A. A. Popov, V. M. Senyavin, M. V. Korobov. Sublimation of hydrofullerenes C60H36 and C60H18. Chemical Physics Letters. Vol.336, No.1-2, 39-46, 2001.

[25] F. Cataldo, D. A. Garcia-Hernandez, A. Manchado, S. Iglesias-Groth. Spectroscopy of fullerenes, fulleranes and PAHs in the UV, visible and near infrared spectral range. Eds. J. Cami, N. Cox, The Diffuse Insterstellar Bands Proceedings IAU Symposium, No.297, 2013.

[26] K. Tokunaga. Hydrogenation of Fullerene C60: Material Design of Organic Semiconductors by Computation. In: Hydrogenation, Ed. I. Karamé, InTech, Chapter 13, 309-326, 2012.

[27] F. Cataldo, S. Iglesias-Groth, D. A. Garcia-Hernandez, A. Manchado. Determination of the Integrated Molar Absorptivity and Molar Extinction Coefficient of Hydrogenated Fullerenes, Fullerenes, Nanotubes, and Carbon Nanostructures, Vol.21, 417-428, 2013.

[28] S. M. Luzan, Y. O. Tsybin, A. V. Talyzin. Reaction of C60 with Hydrogen Gas: In Situ Monitoring and Pathways, The Journal of Physical Chemistry C, Vol.115, No.23, 11484-11492, 2011.

[29] S. Iglesias-Groth, D. A. García-Hernández, F. Cataldo, A. Manchado. Infrared spectroscopy of hydrogenated fullerenes (fulleranes) at extreme temperatures, Monthly Notices of the Royal Astronomical Society, Vol.423, No.3, 2868-2878, 2012.

[30] S. M. Luzan, A. V. Talyzin. Effect of Catalysts on the Reaction of C60 with Hydrogen. Fullerenes, Nanotubes, and Carbon Nanostructures, Vol.20, No.4-7, 319-323, 2012.

[31] F. Cataldo, S. Iglesias-Groth, A. Manchado. On the Radical Cation Spectra of Fullerenes and Fulleranes. Part 2: C60H36, $\mathrm{C} 70 \mathrm{H} 38, \mathrm{C} 60 \mathrm{H} 18$ and $\mathrm{C} 60 \mathrm{Hx} / \mathrm{C} 70 \mathrm{Hy}$ Mixture, Fullerenes, Nanotubes and Carbon Nanostructures, Vol.20, No.8, 672-680, 2012. 